sell," says Xiao.

Ou Mao works at Cnano Technology, a company with corporate headquarters in Santa Clara, California, that mass-produces carbon nanotubes and graphene using techniques developed by chemical engineers at Tsinghua University in 2001. The company now has an annual revenue of more than $\$ 50$ million. Mao has more than 20 years' experience in industrial $R \& D$, and says that the team has focused on ensuring that its products suit the market: "We know that carbon nanotubes have many potential applications across all industries, but it may take many years for industries to accept these novel materials, both in terms of technology and regulations. So we have to be smart and selective in choosing our target market and applications."

The CGIA put together a database of graphene-related projects, categorized by their development stage: lab, pilot or commercialization. Projects at each stage receive a different type of support. Lab projects are tracked and given an incubator when ready; at the pilot stage, the CGIA helps to find investment; and at the commercialization stage, it invites project teams to present their work in front of potential investors and government representatives. The alliance has an annual operating budget of 8 million yuan.

Xiao points to advances in areas such as flexible display screens as examples of successful product development: "We have three members - Chongqing Graphene Technology, Wuxi Electronics and Instruments, and $2 \mathrm{D}$ Carbon Tech - all with the ability to industrially produce graphene flexible screens, and are negotiating cooperations with well-known Chinese electrical appliance manufacturers.'

The next stage for the CGIA is to tackle one of the industry's biggest problems which, as Xiao puts it, is that "the quality of graphene products is totally uncontrollable". In 2016, he therefore helped to launch the China International Graphene Industry Union, which is developing the country's first set of standards. He is also discussing how to reduce technical barriers to trade and joint $\mathrm{R} \& \mathrm{D}$ with international teams such as the International Electrotechnical Commission in São Paulo, Brazil.

"Right now we are working on setting up an international standardization evaluation committee. I think this will help us to speed up the technology transfer from lab to industry," Xiao says. What China's materials science needs now, he adds, is exposure to the rest of the world's scientists. "We need to work on this together as a global group. You cannot just close your door to working on something. People need to know what you're doing." -

Sarah O'Meara is a freelance science writer in London. Additional research by Kevin Schoenmakers. and

\section{Pressure chemist}

Chemist Haiyan Zheng works for the Center for High Pressure Science and Technology Advanced Research (HPSTAR) in Beijing. Nature talks to her about her work at the institute and about equality in science in China.

How did you come to work at HPSTAR? After completing my $\mathrm{PhD}$ in organic chemistry at the Chinese Academy of Sciences Institute of Chemistry in Beijing, I moved to Rice University in Houston, Texas, to study carbon nanomaterials for the purposes of drug delivery. At the same time, my husband was doing his postdoc at the Carnegie Institution for Science in Washington DC under Ho-Kwang Mao, who founded HPSTAR. He introduced me to his research. The following year, I joined Mao's group as a postdoc and began learning about high-pressure technology, and in 2014 came to work at HPSTAR as a staff scientist.

\section{Why did you change your research field?}

High-pressure chemistry is an unexplored area of research, which I really feel needs to be investigated. It's an amazing opportunity for a researcher. Changing my field also meant a chance to work near my husband, which was good for our home life.

\section{What are you working on?}

Everyone at HPSTAR works on different areas of high-pressure science and technology. My job is to better understand how basic organic chemicals react under extreme conditions, including high pressure and high temperatures. In theory, this research could lead to the creation of compounds or carbon structures with new properties.

How much has your scientific focus changed? I still focus on chemistry, just using a different tool. I would now describe my work as physical chemistry. At HPSTAR, we have chemists, physicists and materials scientists all working together to better understand the relationship between the structure of materials and their properties.

\section{What is the goal of your work?}

My team and I would like to understand precisely how chemical reactions happen under extreme conditions, then design molecules

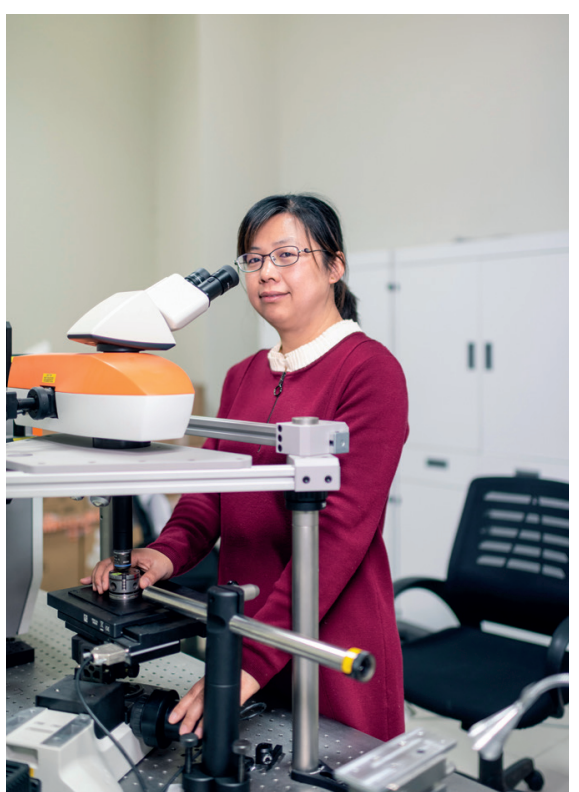

that will be compressed under high pressure and produce the desired product. That's our dream.

What are the obstacles for Chinese women in science?

Your early thirties are a very important time for the development of your career, but it's also the time when women have children. This can cause difficulty, because women can't focus all their energy on work. I'm very lucky. My husband works in the same institute, so that helps us to balance our family and work lives more efficiently. We can discuss our work at any time. Our parents also help us a lot with the care of our four-year-old son.

\section{What does a female scientist need to succeed?}

To be a successful women in science, you need three things: self-motivation, equal opportunities at work and family support. You also need to have a work environment that supports you, and people to help you with family life.

\section{Do women in science receive additional support in China?}

The government tries its best to support women in science. In 2011, the National Natural Science Foundation of China committee increased the age limit for women applying to its Young Scientist Fund from 35 to 40. In the past, everyone had to apply before they reached 36. That's hard for women because of their responsibility for children around that time. After the age limit increased, the number of successful applications from women increased by ten percentage points. I think that it has been a great help for women in their careers.

\section{INTERVIEW BY SARAH O'MEARA}

This interview has been edited for length and clarity. 